OPEN ACCESS

Edited by:

Chiara Nicolazzo,

Sapienza University of Rome, Italy

Reviewed by:

Veronica Aran,

Instituto Estadual do Cérebro Paulo

Niemeyer (IECPN), Brazil

Jean-Christophe Sabourin,

Centre Hospitalier Universitaire (CHU)

de Rouen, France

${ }^{*}$ Correspondence:

Emerik Osterlund

emerik.osterlund@igp.uu.se; emerik.osterlund@gmail.com

${ }^{\dagger}$ These authors have contributed equally to this work and share last authorship

Specialty section:

This article was submitted to Molecular and Cellular Oncology,

a section of the journal

Frontiers in Oncology

Received: 30 November 2021 Accepted: 17 January 2022

Published: 16 February 2022

Citation:

Osterlund E, Ristimäki A, Kytölä $S$,

Kuopio T, Heervä E, Muhonen T,

Halonen $P$, Kallio $R$, Soveri $L M$,

Sundström J, Keinänen M, Ålgars A,

Ristamäki $R$, Sorbye $H$, Pfeiffer $P$,

Nunes L, Salminen T, Lamminmäki $A$,

Mäkinen MJ, Sjöblom T, Isoniemi $H$,

Glimelius B and Osterlund $P$ (2022)

KRAS-G12C Mutation in One

Real-Life and Three Population-

Based Nordic Cohorts of

Metastatic Colorectal Cancer.

Front. Oncol. 12:826073.

doi: 10.3389/fonc.2022.826073

\section{KRAS-G12C Mutation in One Real- Life and Three Population-Based Nordic Cohorts of Metastatic Colorectal Cancer}

Emerik Osterlund ${ }^{\text {* }}$ Ari Ristimäki 2,3 Soili Kytölä 4,5 ${ }^{20 i j o ~ K u o p i o ~ 6,7, ~ E e t u ~ H e e r v a, ~}$ Timo Muhonen ${ }^{10,11}$, Päivi Halonen ${ }^{11,12}$, Raija Kallio ${ }^{13,14}$, Leena-Maija Soveri ${ }^{12,15}$, Jari Sundström ${ }^{16,17}$, Mauri Keinänen ${ }^{18}$, Annika Ålgars ${ }^{8,9}$, Raija Ristamäki ${ }^{8,9}$, Halfdan Sorbye ${ }^{19,20}$, Per Pfeiffer ${ }^{21,22}$, Luís Nunes ${ }^{1}$, Tapio Salminen ${ }^{23,24}$ Annamarja Lamminmäki ${ }^{25,26}$, Markus J. Mäkinen ${ }^{27,28}$, Tobias Sjöblom ${ }^{1}$, Helena Isoniemi ${ }^{29,30 \dagger}$, Bengt Glimelius ${ }^{1 \dagger}$ and Pia Osterlund ${ }^{11,12,23,24,31,32 \dagger}$

${ }^{1}$ Department of Immunology, Genetics and Pathology, Uppsala University, Uppsala, Sweden, 2 Department of Pathology, HUSLAB, HUS Diagnostic Center, Helsinki University Hospital, Helsinki, Finland, ${ }^{3}$ Applied Tumor Genomics Research Program, Research Programs Unit, Faculty of Medicine, University of Helsinki, Helsinki, Finland, ${ }^{4}$ Department of Genetics, HUSLAB, HUS Diagnostic Center, Helsinki University Hospital, Helsinki, Finland, ${ }^{5}$ Department of Genetics, University of Helsinki, Helsinki, Finland, ${ }^{6}$ Department of Pathology, Central Finland Hospital Nova, Jyväskylä, Finland, ${ }^{7}$ Department of Biological and Environmental Science, University of Jyväskylä, Jyväskylä, Finland, ${ }^{8}$ Department of Oncology, Turku University Hospital, Turku, Finland, ${ }^{9}$ Department of Oncology, University of Turku, Turku, Finland, ${ }^{10}$ Department of Oncology, South Carelia Central Hospital, Lappeenranta, Finland, ${ }^{11}$ Department of Oncology, University of Helsinki, Helsinki, Finland, ${ }^{12}$ Department of Oncology, Helsinki University Hospital, Helsinki, Finland, ${ }^{13}$ Department of Oncology, Oulu University Hospital, Oulu, Finland, ${ }^{14}$ Department of Oncology, University of Oulu, Oulu, Finland, ${ }^{15}$ Home Care, Geriatric Clinic and Palliative Care, Joint Municipal Authority for Health Care and Social Services in Keski-Uusimaa, Hyvinkää, Finland, ${ }^{16}$ Department of Pathology, Turku University Hospital, Turku, Finland, 17 Institute of Biomedicine, University of Turku, Turku, Finland, ${ }^{18}$ Department of Genetics, Fimlab Laboratories, Tampere, Finland, ${ }^{19}$ Department of Oncology, Haukeland University Hospital, Bergen, Norway, ${ }^{20}$ Department of Clinical Science, University of Bergen, Bergen, Norway, ${ }^{21}$ Department of Oncology, Odense University Hospital, Odense, Denmark, ${ }^{22}$ Department of Clinical Research, University of Southern Denmark, Odense, Denmark, ${ }^{23}$ Department of Oncology, Tampere University Hospital, Tampere, Finland, ${ }^{24}$ Department of Oncology, University of Tampere, Tampere, Finland, ${ }^{25}$ Department of Oncology, Kuopio University Hospital, Kuopio, Finland, ${ }^{26}$ Department of Medicine, University of Eastern Finland, Kuopio, Finland, ${ }^{27}$ Department of Pathology, Oulu University Hospital, Oulu, Finland, ${ }^{28}$ Department of Pathology, Cancer and Translational Medicine Research Unit, University of Oulu, and Medical Research Center Oulu, Oulu, Finland, ${ }^{29}$ Department of Transplantation and Liver Surgery, Helsinki University Hospital, Helsinki. Finland, ${ }^{30}$ Department of Surgery, University of Helsinki. Helsinki, Finland, ${ }^{31}$ Department of Gastrointestinal Oncology, Karolinska Universitetssjukhuset, Stockholm, Sweden, ${ }^{32}$ Department of Oncology/Pathology, Karolinska Institutet, Stockholm, Sweden

Background: KRAS mutations, present in over $40 \%$ of metastatic colorectal cancer ( $\mathrm{mCRC})$, are negative predictive factors for anti-EGFR therapy. Mutations in KRAS-G12C have a cysteine residue for which drugs have been developed. Published data on this specific mutation are conflicting; thus, we studied the frequency and clinical characteristics in a real-world and population-based setting.

Methods: Patients from three Nordic population-based cohorts and the real-life RAXOstudy were combined. RAS and BRAF tests were performed in routine healthcare, except for one cohort. The dataset consisted of 2,559 patients, of which 1,871 could be accurately classified as KRAS, NRAS, and BRAF-V600E. Demographics, treatments, and outcomes were compared using logistic regression. Overall survival (OS) was 
estimated with Kaplan-Meier, and differences were compared using Cox regression, adjusted for baseline factors.

Results: The KRAS-G12C frequency was $2 \%-4 \%$ of all tested in the seven cohorts (mean $3 \%$ ) and $4 \%-8 \%$ of KRAS mutated tumors in the cohorts (mean $7 \%$ ). Metastasectomies and ablations were performed more often (38\% vs. 28\%, $p=$ 0.040 ), and bevacizumab was added more often (any line $74 \%$ vs. $59 \%, p=0.007$ ) for patients with KRAS-G12C- vs. other KRAS-mutated tumors, whereas chemotherapy was given to similar proportions. OS did not differ according to KRAS mutation, neither overall (adjusted hazard ratio $(\mathrm{HR}) 1.03 ; 95 \% \mathrm{Cl} 0.74-1.42$, reference KRAS-G12C) nor within treatment groups defined as "systemic chemotherapy, alone or with biologics", "metastasectomy and/or ablations", or "best supportive care", RAS and BRAF wildtype tumors ( $\mathrm{n}=548$ ) differed similarly to KRAS-G12C, as to other KRAS- or NRASmutated $(n=66)$ tumors.

Conclusions: In these real-life and population-based cohorts, there were no significant differences in patient characteristics and outcomes between patients with KRAS-G12C tumors and those with other KRAS mutations. This contrasts with the results of most previous studies claiming differences in many aspects, often with worse outcomes for those with a KRAS-G12C mutation, although not consistent. When specific drugs are developed, as for this mutation, differences in outcome will hopefully emerge.

Keywords: colorectal cancer, metastatic, KRAS mutation, KRAS-G12C mutation, population-based, real-world

\section{INTRODUCTION}

Mutations in the RAS genes are common oncogenic drivers (1). In colorectal cancer (CRC), Kirsten rat sarcoma viral oncogene homolog (KRAS) is mutated $(\mathrm{mt})$ in over $40 \%$ and neuroblastoma RAS viral oncogene homolog (NRAS) in about $4 \%$ (2). The specific mutated codon impacts the activity of the enzyme, and this may impact the clinical behavior of the tumor. Activating missense mutations in RAS cause resistance to epidermal growth factor receptor (EGFR) inhibition, and although different mutations may have somewhat variable resistance $(3,4)$, in practice, all KRAS and NRAS mutations are predictive markers for no benefit of anti-EGFR therapy. It has been challenging to develop therapies directed against KRAS mutated tumors due to multiple different activating mutations in the RAS genes $(5,6)$. The KRAS-G12C has a cysteine residue for which specific drugs, such as sotorasib (AMG 510, Amgen, Thousand Oaks, CA, USA) and adagrasib (MRTX849, Mirati Therapeutics, San Diego, CA, USA), have been developed and clinically explored with promising results $(7,8)$.

The importance of different KRAS mutations on the clinical behavior of metastatic CRC (mCRC) has been explored, and differences have been reported (9). The clinical relevance of KRAS-G12C is unclear (10-17). The frequency of KRAS-G12C has been reported to be $2 \%-8 \%$ in large databases of molecularly analyzed CRCs $(12,14,18,19)$. In hospital-based series, the proportion of KRAS-G12C tumors was $2 \%-4 \%$ of all mCRC tumors $(11,15,16,20)$, whereas greater variability was seen in the proportion of the KRAS-mutated population $(6 \%-17 \%)(11$,
$13,16,17,20-22)$. Sex- and age-related differences and differences in the metastatic pattern have been reported between different KRAS mutations, with no consistent results $(12,13,22)$.

Several studies have explored the relation between progressionfree (PFS) and overall survival (OS) and KRAS mutation type in mCRC. Two Italian hospital-based series reported worse survival for those with KRAS-G12C and KRAS-G12S compared to other KRAS mutations $(13,20)$. In a pooled analysis of three German trials of retrospectively analyzed KRAS codon 12 mutant tumors, patients with G12C (and G12S) mutations fared worse when treated with chemotherapy and EGFR inhibition (23). In a follow-up study including two more trials, the same group found no difference in PFS or OS between different KRAS exon 2 mutations, but KRAS-G12C had the numerically worst median OS (mOS) and was the only mutation that significantly differed from KRAS wild-type (wt) tumors (11). Among 4,632 patients with molecularly profiled tumors at MD Anderson Cancer Center, 134 (3\%) had a KRAS-G12C mutation. When 53 additional patients were added, those with a KRAS-G12C had worse PFS and OS (15). A Japanese study including 1632 patients treated with chemotherapy at 4 hospitals found 45 (6\%) KRAS-G12C tumors among 696 KRAS mutated tumors and similarly found that both PFS and OS were worse for the KRAS-G12C cases compared to those having other KRAS mutations (16). Finally, in yet another Italian hospital-based study, 120 patients with KRAS mutated tumors were treated with a doublet chemotherapy regimen and bevacizumab; 15 (12\%) patients with a KRAS-G12C had a lower response rate than those with another mutation $(27 \%$ vs. $52 \%, \mathrm{p}=$ 0.017); however, no differences in PFS or OS were seen (17). 
The studies performed so far have been restricted to patients included in clinical trials, hospital-based series, or large databases with unknown patient selection. Since certain molecular changes in the background population of patients with mCRC differ substantially from those in clinical trials and hospital series (2426), the representativity of the present knowledge can be discussed. Further, since these mutations are not particularly common, the number of cases is limited in most studies, making estimates uncertain. Extensive knowledge about KRAS-G12C frequency, the possible presence of characteristics differing from other KRAS mutations, and their relation to survival and treatment response is not available. For these reasons, we examined three population-based Nordic patient materials and one large real-life material for the presence of KRAS-G12C and their relations to patient characteristics and outcome compared to those having other RAS and $\mathrm{v}$-Raf murine sarcoma viral oncogene homolog B (BRAF)-V600E mutations.

\section{MATERIALS AND METHODS}

Patients from four Nordic cohorts were included: the prospective real-life Finnish RAXO-study, a population-based data collection cohort of Finnish patients molecularly tested at four hospitals covering all medical care in the surrounding regions (Helsinki, Jyväskylä, Tampere, and Turku), the population-based Scandinavian prospective registration of mCRC (PRCRCstudy), and a Swedish population-based cohort (Uppsala region) (Figure 1 and Supplementary Table $\mathbf{1}$ ).

Inclusion criteria for the RAXO-study $(27,28)$ were patients eligible for first-line chemotherapy with any oncological treatment regimen, age over 18 years, and histologically confirmed CRC with distant metastases or locally advanced primary tumor not curatively treatable. The patients were recruited between 2012 and 2018. In the Finnish populationbased RAXO data-collection cohort (referred to here as the Finnish population cohort), all patients with a diagnosis of mCRC between 2011 and 2018 from four regions (Helsinki, Jyväskylä, Tampere, and Turku, covering $62 \%$ of the Finnish population) were included [the prospective RAXO datacollection protocol and preliminary results from Tampere and Turku have been published (27)]. This cohort $(n=3,953)$ partly overlaps with the real-life RAXO-study $(n=1,060)$ for mutation frequencies; most of the hospitals recruiting patients to the reallife RAXO study were in the four Finnish regions. The exact frequency of KRAS-G12C mutations among all tested tumors could not be calculated since validated comprehensive data of all non-KRAS-G12C cases at these four hospitals are not available; therefore, we have not included detailed characteristics of these patients in the presentation, only the KRAS-G12C cases (see Supplementary Table 2). Duplicate patients with KRAS-G12C tumors from the RAXO-study were removed $(n=23)$ from the Finnish population cohort.

In the PRCRC-study, all patients $(n=798)$ with a diagnosis of incurable metastatic disease from three regions in Norway, Denmark, and Sweden were included between 2003 and
2006 (29). In the Uppsala region cohort, all mCRC patients were prospectively identified in a biobank initiative UppsalaUmeå Comprehensive Cancer Consortium (UCAN)) (30) since April 2010, and the remaining patients with mCRC since January 2010 were retrospectively identified using a hospital-based registry and the Swedish ColoRectal Cancer Registry (SCRCR) (31). After a validation study against the medical records of all patients with a diagnosis of CRC since January 2010, this cohort can be considered 100\% complete (32).

The patient cohorts are presented in Figure 1. To compare characteristics of patients with KRAS-G12C tumors with those other KRAS mutated from the same populations, demographics for the real-life RAXO-study, the PRCRC-study, and Uppsala region cohorts, excluding those identified in the Finnish population cohort (from the four Finnish regions outside the real-life RAXO study), are presented in Supplementary Tables 4, 5 .

The patients were treated according to routine clinical practice during the inclusion periods. Details for the oldest PRCRC cohort have been described in (29). For the other more recent cohorts, the European Society for Medical Oncology (ESMO) guidelines were followed $(33,34)$.

\section{Molecular Analyses}

Testing for the presence of RAS and BRAF mutations and mismatch repair analyses in the real-life RAXO-study, the Finnish population cohort, and the Uppsala region cohort was done in clinical routine (or within a study program for 14 patients in the Uppsala region cohort) using accredited techniques in the majority, as described below. In the Uppsala region, the ambition was to test for these mutations in all patients, whereas the indication to test in the RAXO-study and the Finnish population cohort was planned systemic therapy for mCRC. The techniques used for testing varied through the years. During the first years of inclusion, a pyrosequencing technique was usually performed for Swedish cohorts (35) and in $0.3 \%$ in the RAXO-study, or reverse transcriptase polymerase chain reaction (RT-PCR) for Finnish cohorts (42\% in the RAXOstudy), but Sanger PCR was rarely used $(0.7 \%$ of RAXOstudy). Two-step Biocartis Idylla testing (first KRAS [exons 2-4] testing and if wt then NRAS [exons 2-4] and BRAF [V600E and non-V600E] testing) has been used for 123 (12\%) patients in the RAXO-study at Oulu and Turku University Hospitals. During the latter parts or at least from 2014, a nextgeneration sequencing (NGS) technique was generally used. The composition of the NGS panels varied between the hospitals and through the years but always contained analyses, allowing testing of the presence of hotspot mutations in extended $R A S$, including both KRAS and NRAS exons 2-4 (codons 12, 13, 59, 61, 117, and 146) and for the BRAF-V600E mutation according to ESMO recommendations (34). Routine NGS testing was performed in the molecular pathology units at Uppsala, Helsinki, Tampere, Jyväskylä, Charité, and Nijmegen University Hospitals. In the PRCRC-study cohort, these analyses were performed using an NGS panel as described in (26). All patients with at least KRAS exon 2 testing were included in frequency analyses, presented as KRAS-G12C rate of all tested tumors and of patients with any KRAS mutation. 


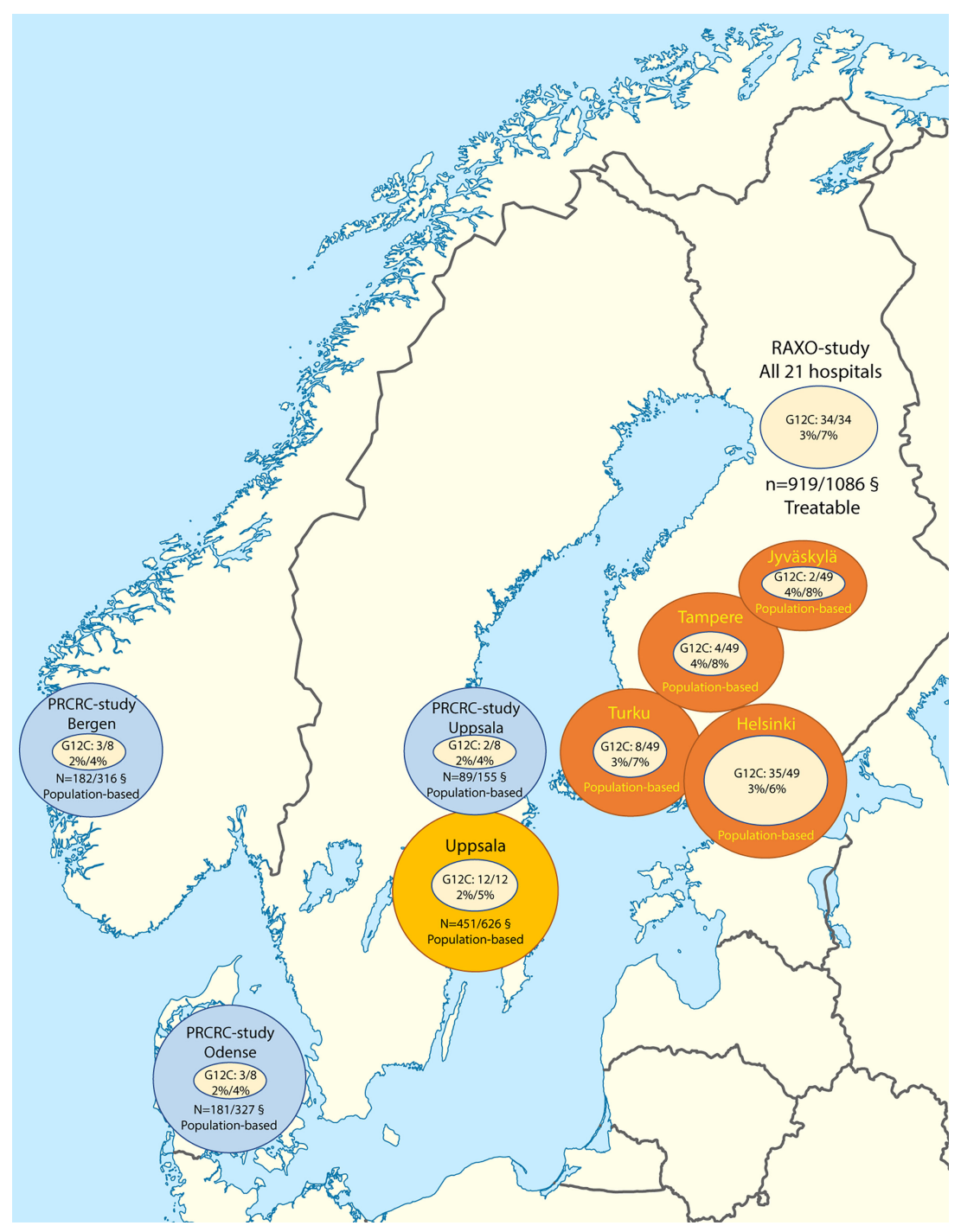

FIGURE 1 | Patient inclusion in the Nordic cohorts including KRAS-G12C mutation rates per all tested (at least KRAS) tumors and among all KRAS-mutated tumors. ${ }^{\S}$ Adequately grouped KRAS, NRAS, and BRAF-V600E status/study patients.

Patients that could not be accurately classified for KRAS, NRAS, and $B R A F$ were excluded from demographics presentation and survival analyses (Figure 1 and Supplementary Tables 1, 2). To be classified as adequately analyzed, the presence of a KRAS or $B R A F-V 600 \mathrm{E}$ mutation, assuming that these mutations are mutually exclusive, was sufficient. Before a tumor was considered RAS and BRAF wt, testing of KRAS exons 2-4, NRAS exons 2-4, and BRAF-V600E was required. In 428 (17\%) of 2,559 patients, no molecular tests had been performed, and 260 (10\%) could not be adequately characterized (due to missing $K R A S, N R A S$, or BRAF analyses in 229, atypical non-BRAFV600E mutations in 24, and multiple mutations in 7 [KRAS and NRAS $(\mathrm{n}=6)$ and KRAS-G12C and BRAF-V600E $(\mathrm{n}=1)]$. OS is presented separately for the tested but not adequately characterized group.

\section{Statistical Analyses}

Demographics, treatments, and outcomes between KRAS-G12C and other KRAS mutations were compared with logistic regression models and with chi-square for cohorts. The MannWhitney test was used to compare non-normally distributed variables. Survival was estimated with the Kaplan-Meier estimate and compared using the log-rank test and Cox regression. OS was also adjusted for age, sex, Eastern Cooperative Oncology Group (ECOG) performance status, primary tumor site, presentation and number of metastatic sites, treatment groups, and cohort. KRAS-G12C was used as a reference in these analyses. Two-tailed p-values of $<0.05$ and $95 \%$ CI not crossing 1 were considered statistically significant. All statistical analyses were performed using SPSS Statistics version 25 and 27. 


\section{Clinical Trial Identification and Ethical Permission}

Clinical trial identification is NCT01531595 and EudraCT 2011003137-33 for the RAXO-study and Finnish population cohort. Ethical permission was obtained for all collections by the ethical committees at the Helsinki University Hospital, Haukeland University Hospital, Odense University Hospital, and Uppsala University. These permissions included retrospective identification of all patients with a diagnosis of mCRC living in the catchment areas of the Scandinavian PRCRC cohort and in the Uppsala region at the time of diagnosis of their primary tumor and to perform the molecular analyses of their tumors. All studies were conducted in accordance with the Declaration of Helsinki.

\section{RESULTS}

\section{Characteristics of All and Tested Patients in the Cohorts}

The characteristics of all 2,559 patients in the cohorts are presented in Supplementary Table 1. Patients in the PRCRCstudy were slightly older, were more likely female, had more right-sided colon cancers, and were cared for without any tumor controlling therapy (best supportive care (BSC)) more often than those in the more recent Uppsala region and RAXO-study cohorts. Patients in the real-life RAXO-study were younger, had better ECOG performance status, and more often received active tumor-controlling therapy (according to inclusion criteria), especially metastasectomies and/or local ablative therapy (LAT). mOS of all patients in the cohorts was the shortest in the oldest PRCRC-study cohort, intermediate in the recent population-based cohorts [Uppsala region 15.3 months, Supplementary Table 1, Tampere region 16 months, and Turku region 16 months, data shown in (27)], and the longest in the real-life RAXO-study. The OS differences were much smaller when analyzed per treatment group, i.e., in patients receiving cytotoxics only, cytotoxics combined with anti-vascular endothelial growth factor (anti-VEGF) or anti-EGFR, and metastasectomy and/or LAT (such as thermoablation and stereotactic radiotherapy) (Supplementary Table 1).

Of all molecularly tested (at least KRAS exon 2 analyzed) tumors in the cohorts, between $2 \%$ and $4 \%$, with a mean of $3 \%$, had a mutation in KRAS-G12C (PRCRC-study 2\%, Uppsala region $2 \%$, Helsinki region $3 \%$, real-life RAXO-study $3 \%$, Turku region $3 \%$, Jyväskylä region $4 \%$, and Tampere region 4\%; Figure 1).

The proportion of a KRAS-G12C mutation among the tumors with a KRAS mutation was between $4 \%$ and $8 \%$, with a mean of $7 \%$ (PRCRC-study 4\%, Uppsala region 5\%, Helsinki region 6\%, RAXO-study $7 \%$, Turku region $7 \%$, Jyväskylä region $8 \%$, and Tampere region 8\%; Figure 1). KRAS-G12C was mutually exclusive for all but one patient (with $B R A F-V 600 \mathrm{Emt}$ ).

\section{Characteristics of Molecularly Accurately Grouped Patients}

The characteristics of all 1,871 patients accurately characterized as KRAS, NRAS, and BRAF-V600E from the four cohorts including the 49 unique Finnish population cohort patients identified with a KRAS-G12C in the four hospital regions in Finland are presented in Figure 1 and Supplementary Table 2. Supplementary Table $\mathbf{2}$ shows that patients accurately analyzed for KRAS, NRAS, and BRAF were more similar than all patients in the cohorts were (presented in Supplementary Table 1). The proportions of the different treatment groups differed between the cohorts, and OS accordingly. The OS did not differ between cohorts in the treatment groups, i.e., cytotoxics only, cytotoxics combined with anti-VEGF/anti-EGFR, or metastasectomy and/ or LAT (Supplementary Table 2). The BSC group had the shortest OS in all cohorts, although it was longer in the population cohorts compared to the real-life RAXO study.

The rates of different mutations in accurately molecularly analyzed patients are shown in Supplementary Table 2. The proportion of tumors with a KRAS and NRAS mutation was rather similar between the cohorts. The most striking difference between the cohorts was in the proportion of BRAF-V600Emutated tumors: $20 \%$ in the PRCRC-study, $20 \%$ in the Uppsala region, $18 \%$ in the Finnish population cohort, and $10 \%$ in the real-life RAXO-study.

The characteristics of the patients with the different RAS and BRAF-V600E mutations are shown in Table $\mathbf{1}$ and Supplementary Table 3. The same characteristics for the patients excluding the 49 patients from the Finnish population cohorts are shown in Supplementary Table 4. No differences were observed between the patients with KRAS-G12C ( $\mathrm{n}=103)$ or other KRAS mutations $(\mathrm{n}=881)$ according to age, sex, performance status, smoking status, synchronous/metachronous presentation, primary tumor site, degree of differentiation, histology, number of metastatic sites, metastatic location, blood counts, alkaline phosphatase, or carcinoembryonic antigen levels. KRAS mutated tumors were seldom associated with deficient mismatch repair (dMMR) status (3\% for both KRAS-G12C and other KRASmt), however, based upon a few cases; in contrast, dMMR was associated with a BRAF-V600E mutation much more frequently (29\%). For KRAS-G12C vs. other KRAS mutations, the primary tumor sites were the right colon in $28 \%$ vs. $34 \%$, the left colon in $35 \%$ vs. $30 \%$, and the rectum in $37 \%$ vs. $36 \%$. Liver metastases were present in $64 \%$ vs. $70 \%$, lung in $39 \%$ vs. $36 \%$, distant lymph nodes in $24 \%$ vs. $23 \%$, and peritoneal in $15 \%$ vs. $19 \%$ of KRASG12C compared with other KRAS mutations.

\section{Treatments and Outcome}

Treatments provided for all accurately molecularly analyzed patients are shown in Table $\mathbf{2}$ and Supplementary Table 5. Systemic therapy was given to $85 \%$ vs. $84 \%$ of KRAS-G12C vs. other KRAS mutant cases, with no differences in the number of lines of therapy, treatment responses, and drug exposures apart from more anti-VEGF use in any line (but not first line) for patients with a KRAS-G12C mutation.

Metastasectomy and LAT were performed in $38 \%$ vs. $28 \%$ for KRAS-G12C vs. other KRAS mutant cases $(\mathrm{p}=0.042)$. This did not have a significant effect on OS (median 26.2 vs. 22.0 months; adjusted hazard ratio (HR) 1.03; 95\% CI 0.75-1.43, with KRASG12C as the reference; unadjusted HR shown in Figure 2A). 
TABLE 1 | Demographics according to the type of mutation.

\begin{tabular}{|c|c|c|c|c|c|c|c|c|c|c|c|c|c|c|}
\hline \multirow[b]{2}{*}{ Total } & & \multicolumn{2}{|c|}{ All } & \multicolumn{2}{|c|}{$\begin{array}{c}\text { KRAS-G12C } \\
\mathrm{mt}\end{array}$} & \multicolumn{2}{|c|}{$\begin{array}{c}\text { Other KRAS } \\
\text { mt }\end{array}$} & \multicolumn{2}{|c|}{ NRAS mt } & \multicolumn{2}{|c|}{$\begin{array}{c}R A S \text { and } B R A F \\
\text { wt }\end{array}$} & \multicolumn{2}{|c|}{$\begin{array}{c}\text { BRAF-V600E } \\
\mathrm{mt}\end{array}$} & \multirow[t]{2}{*}{ p-Value* } \\
\hline & & 1,871 & $100 \%$ & 103 & $100 \%$ & 881 & $100 \%$ & 66 & $100 \%$ & 548 & $100 \%$ & 273 & $100 \%$ & \\
\hline Median age (range) & & 68 & (22-99) & 67 & (35-86) & 69 & (23-99) & 64 & $(30-87)$ & 67 & $(22-95)$ & 70 & (33-90) & 0.036 \\
\hline \multirow[t]{2}{*}{ Age groups } & $\leq 70$ & 1,074 & $57 \%$ & 63 & $61 \%$ & 484 & $55 \%$ & 44 & $67 \%$ & 350 & $64 \%$ & 137 & $50 \%$ & ref \\
\hline & $>70$ & 797 & $43 \%$ & 40 & $39 \%$ & 397 & $45 \%$ & 22 & $33 \%$ & 198 & $36 \%$ & 136 & $50 \%$ & 0.230 \\
\hline \multirow[t]{2}{*}{ Sex } & Female & 818 & $44 \%$ & 46 & $45 \%$ & 378 & $43 \%$ & 28 & $42 \%$ & 198 & $36 \%$ & 168 & $62 \%$ & ref \\
\hline & Male & 1,053 & $56 \%$ & 57 & $55 \%$ & 503 & $57 \%$ & 38 & $58 \%$ & 350 & $64 \%$ & 105 & $38 \%$ & 0.734 \\
\hline \multirow[t]{4}{*}{ ECOG Performance status } & 0 & 593 & $32 \%$ & 27 & $26 \%$ & 297 & $34 \%$ & 25 & $38 \%$ & 176 & $32 \%$ & 68 & $25 \%$ & ref \\
\hline & 1 & 815 & $44 \%$ & 50 & $49 \%$ & 394 & $45 \%$ & 31 & $47 \%$ & 241 & $44 \%$ & 99 & $37 \%$ & 0.184 \\
\hline & $2-4$ & 457 & $25 \%$ & 25 & $25 \%$ & 188 & $21 \%$ & 10 & $15 \%$ & 130 & $24 \%$ & 104 & $38 \%$ & 0.194 \\
\hline & Missing & 6 & - & 1 & - & 2 & - & 0 & - & 1 & - & 2 & - & - \\
\hline \multirow[t]{4}{*}{ Primary tumor } & Right colon & 624 & $34 \%$ & 29 & $28 \%$ & 295 & $34 \%$ & 9 & $14 \%$ & 95 & $17 \%$ & 196 & $73 \%$ & ref \\
\hline & Left colon & 619 & $33 \%$ & 36 & $35 \%$ & 266 & $30 \%$ & 26 & $39 \%$ & 241 & $44 \%$ & 50 & $19 \%$ & 0.225 \\
\hline & Rectum & 612 & $33 \%$ & 38 & $37 \%$ & 312 & $36 \%$ & 31 & $47 \%$ & 207 & $38 \%$ & 24 & $9 \%$ & 0.409 \\
\hline & Multiple/unknown & 16 & - & 0 & - & 8 & - & 0 & - & 5 & - & 3 & - & - \\
\hline \multirow[t]{3}{*}{ Differentiation } & Well/moderate & 1,194 & $79 \%$ & 74 & $85 \%$ & 588 & $84 \%$ & 47 & $86 \%$ & 363 & $81 \%$ & 122 & $53 \%$ & ref \\
\hline & Poor/undifferentiated & 325 & $21 \%$ & 13 & $15 \%$ & 111 & $16 \%$ & 8 & $15 \%$ & 85 & $19 \%$ & 108 & $47 \%$ & 0.821 \\
\hline & Missing & 352 & - & 60 & - & 182 & - & 11 & - & 100 & - & 43 & - & - \\
\hline \multirow[t]{2}{*}{ Presentation of metastases } & Synchronous & 1,147 & $61 \%$ & 61 & $59 \%$ & 555 & $63 \%$ & 41 & $62 \%$ & 318 & $58 \%$ & 172 & $63 \%$ & ref \\
\hline & Metachronous & 724 & $39 \%$ & 42 & $41 \%$ & 326 & $37 \%$ & 25 & $38 \%$ & 230 & $42 \%$ & 101 & $37 \%$ & 0.454 \\
\hline \multirow[t]{3}{*}{ Number of metastatic sites } & 1 & 916 & $49 \%$ & 54 & $52 \%$ & 443 & $50 \%$ & 31 & $47 \%$ & 263 & $48 \%$ & 125 & $46 \%$ & ref \\
\hline & 2 & 615 & $33 \%$ & 33 & $32 \%$ & 287 & $33 \%$ & 22 & $33 \%$ & 178 & $32 \%$ & 95 & $35 \%$ & 0.803 \\
\hline & $3+$ & 340 & $18 \%$ & 16 & $16 \%$ & 151 & $17 \%$ & 13 & $20 \%$ & 107 & $20 \%$ & 53 & $19 \%$ & 0.640 \\
\hline \multirow[t]{6}{*}{ Metastatic sites } & Liver & 1,289 & $69 \%$ & 66 & $64 \%$ & 615 & $70 \%$ & 51 & $77 \%$ & 412 & $75 \%$ & 145 & $53 \%$ & 0.234 \\
\hline & Lung & 587 & $31 \%$ & 40 & $39 \%$ & 321 & $36 \%$ & 22 & $33 \%$ & 136 & $25 \%$ & 68 & $25 \%$ & 0.633 \\
\hline & Lymph nodes & 499 & $27 \%$ & 25 & $24 \%$ & 199 & $23 \%$ & 18 & $27 \%$ & 152 & $28 \%$ & 105 & $38 \%$ & 0.700 \\
\hline & Peritoneum & 388 & $21 \%$ & 15 & $15 \%$ & 169 & $19 \%$ & 11 & $17 \%$ & 104 & $19 \%$ & 89 & $33 \%$ & 0.257 \\
\hline & Bone & 69 & $4 \%$ & 3 & $3 \%$ & 31 & $4 \%$ & 2 & $3 \%$ & 23 & $4 \%$ & 10 & $4 \%$ & 0.750 \\
\hline & Other & 257 & $14 \%$ & 15 & $15 \%$ & 106 & $12 \%$ & 9 & $14 \%$ & 78 & $14 \%$ & 49 & $18 \%$ & 0.460 \\
\hline \multirow[t]{3}{*}{ MMR-status } & pMMR & 908 & $92 \%$ & 33 & $97 \%$ & 425 & $97 \%$ & 27 & $90 \%$ & 292 & $98 \%$ & 131 & $71 \%$ & ref \\
\hline & $\mathrm{dMMR}$ & 77 & $8 \%$ & 1 & $3 \%$ & 12 & $3 \%$ & 3 & $10 \%$ & 7 & $2 \%$ & 54 & $29 \%$ & 0.947 \\
\hline & Missing & 886 & - & 69 & - & 444 & - & 36 & - & 249 & - & 88 & - & - \\
\hline
\end{tabular}

dMMR, deficient mismatch repair; ECOG, Eastern Cooperative Oncology Group; MMR, mismatch repair; pMMR, proficient mismatch repair; PS, performance status.

${ }^{*} p$-Value between KRAS-G12C mt and Other KRAS mt. Significant differences in bold.

OS within each separate treatment group (metastasectomy/LAT, "systemic therapy only" and BSC alone) did not differ (Figures 2B-D and Supplementary Figure 1 for treatment groups in KRAS-G12C tumors only). Median PFS in patients treated with "systemic therapy only" was 12.7 months in KRASG12C tumors and 11.7 months in other KRAS mutations, with adjusted HR 1.09 (95\% CI 0.71-1.65) (Supplementary Figure 3A). OS and PFS excluding the 49 patients from the Finnish population cohort are presented in Supplementary Figures 2A-D, 3B.

\section{Characteristics of Patients With a KRASG12C Mutation in Comparison With Those With Other RAS and BRAF Mutations}

RAS and BRAF wt tumors revealed the same associations for KRAS-G12C as for other KRAS or NRAS mutations. Patients with tumors being RAS and BRAF wt were right-sided less often (Table 1). Patients with these tumors also had the longest OS (median 29.6 vs. 26.2 months for KRAS-G12C and 22.0 months for other KRASmt (Figure 2A). Median PFS in patients treated with "systemic chemotherapy only" was longer in RAS and BRAF wt tumors (10.6 months) than in KRAS-G12C tumors (10.1 months), other KRASmt (9.8 months), or NRASmt (9.4 months)
(Supplementary Figure 3A). mOS in the patients not adequately classified as RAS and BRAF wt (or with double mutations) was 22.6 months.

$B R A F-V 600 \mathrm{E}$ cases differed in many aspects from the other mutations and more often had progressive disease (PD) as the best response to chemotherapy, a surgical procedure was done less often, and the poorest PFS and OS were seen, regardless of treatment group (Table 1, Figures 2A-D, Supplementary Tables 3, 4, and Supplementary Figures 2, 3).

\section{DISCUSSION}

In these real-life and population-based cohorts, mutations in KRAS-G12C were seen in $2 \%-4 \%$ of all tumors in patients with mCRC; actually, the proportion was the lowest in the population-based cohorts, with $2 \%-3 \%$, and up to $4 \%$ when analyzed in the real-life RAXO-study and Finnish population cohorts, where most of the patients could be actively treated. The proportion of this mutation among KRAS mutated tumors was $4 \%-8 \%$, similarly the lowest in the population-based cohorts $(4 \%$ in the PRCRC-study and Uppsala region), proportions that are considerably lower than in all so far reported hospital-based or clinical trial series $(6 \%-17 \%)(11,13,15,16,20,21)$, but in line 
TABLE 2 | Treatment according to the type of mutation.

\begin{tabular}{|c|c|c|c|c|c|c|c|c|c|c|c|c|c|c|}
\hline \multirow[b]{2}{*}{ Total } & & \multicolumn{2}{|c|}{ All } & \multicolumn{2}{|c|}{$\begin{array}{c}\text { KRAS-G12C } \\
\mathrm{mt}\end{array}$} & \multicolumn{2}{|c|}{$\begin{array}{c}\text { Other KRAS } \\
\mathrm{mt}\end{array}$} & \multirow[t]{2}{*}{ p-Value* } & \multicolumn{2}{|c|}{ NRAS mt } & \multicolumn{2}{|c|}{$\begin{array}{c}R A S \text { and } B R A F \\
\text { wt }\end{array}$} & \multicolumn{2}{|c|}{$\begin{array}{c}\text { BRAF V600E } \\
\mathrm{mt}\end{array}$} \\
\hline & & 1,871 & $100 \%$ & 103 & $100 \%$ & 881 & $100 \%$ & & 66 & $100 \%$ & 548 & $100 \%$ & 273 & $100 \%$ \\
\hline \multirow[t]{3}{*}{ Type of treatment } & Systemic therapy & 1,077 & $58 \%$ & 51 & $50 \%$ & 510 & $58 \%$ & ref & 39 & $59 \%$ & 308 & $56 \%$ & 169 & $62 \%$ \\
\hline & Metastasectomy and/or LAT & 516 & $28 \%$ & 39 & $38 \%$ & 246 & $28 \%$ & 0.042 & 22 & $33 \%$ & 180 & $33 \%$ & 29 & $11 \%$ \\
\hline & Best supportive care & 278 & $15 \%$ & 13 & $13 \%$ & 125 & $14 \%$ & 0.904 & 5 & $8 \%$ & 60 & $11 \%$ & 75 & $27 \%$ \\
\hline Total chemotherapy & & 1,558 & $100 \%$ & 88 & $100 \%$ & 743 & $100 \%$ & & 59 & $100 \%$ & 476 & $100 \%$ & 192 & $100 \%$ \\
\hline \multirow[t]{3}{*}{ Number of lines } & 1 & 625 & $40 \%$ & 32 & $36 \%$ & 291 & $39 \%$ & ref & 21 & $36 \%$ & 197 & $41 \%$ & 84 & $44 \%$ \\
\hline & 2 & 433 & $28 \%$ & 27 & $31 \%$ & 204 & $27 \%$ & 0.503 & 14 & $24 \%$ & 115 & $24 \%$ & 73 & $38 \%$ \\
\hline & $\geq 3$ & 500 & $32 \%$ & 29 & $33 \%$ & 248 & $33 \%$ & 0.820 & 24 & $41 \%$ & 164 & $34 \%$ & 35 & $18 \%$ \\
\hline \multirow[t]{5}{*}{ First line chemotherapy } & 5-FU & 1,519 & $97 \%$ & 86 & $98 \%$ & 729 & $98 \%$ & 0.802 & 57 & $97 \%$ & 462 & $97 \%$ & 185 & $96 \%$ \\
\hline & Oxaliplatin & 913 & $59 \%$ & 48 & $55 \%$ & 453 & $61 \%$ & 0.245 & 33 & $56 \%$ & 268 & $56 \%$ & 111 & $58 \%$ \\
\hline & Irinotecan & 414 & $27 \%$ & 26 & $30 \%$ & 165 & $22 \%$ & 0.124 & 17 & $29 \%$ & 153 & $32 \%$ & 53 & $28 \%$ \\
\hline & Bevacizumab & 670 & $43 \%$ & 47 & $53 \%$ & 348 & $47 \%$ & 0.244 & 33 & $56 \%$ & 163 & $34 \%$ & 79 & $41 \%$ \\
\hline & anti-EGFR & 164 & $11 \%$ & 1 & $1 \%$ & 12 & $2 \%$ & 0.734 & 4 & $7 \%$ & 136 & $29 \%$ & 11 & $6 \%$ \\
\hline \multirow[t]{4}{*}{ Best response in first line } & PR/CR/NED & 822 & $57 \%$ & 47 & $57 \%$ & 368 & $53 \%$ & 0.579 & 32 & $58 \%$ & 307 & $68 \%$ & 68 & $40 \%$ \\
\hline & $\mathrm{SD}$ & 428 & $30 \%$ & 23 & $28 \%$ & 238 & $35 \%$ & ref & 14 & $25 \%$ & 97 & $21 \%$ & 56 & $33 \%$ \\
\hline & PD & 198 & $14 \%$ & 13 & $16 \%$ & 83 & $12 \%$ & "، & 9 & $16 \%$ & 48 & $11 \%$ & 45 & $27 \%$ \\
\hline & Missing & 110 & - & 5 & - & 54 & - & - & 4 & - & 24 & - & 23 & - \\
\hline \multirow[t]{5}{*}{ Chemotherapy all lines } & $5-F U$ & 1,525 & $98 \%$ & 87 & $99 \%$ & 731 & $98 \%$ & 0.734 & 57 & $97 \%$ & 463 & $97 \%$ & 187 & $97 \%$ \\
\hline & Oxaliplatin & 1,193 & $77 \%$ & 67 & $76 \%$ & 581 & $78 \%$ & 0.659 & 47 & $80 \%$ & 355 & $75 \%$ & 143 & $74 \%$ \\
\hline & Irinotecan & 1,088 & $70 \%$ & 61 & $69 \%$ & 511 & $69 \%$ & 0.917 & 46 & $78 \%$ & 344 & $72 \%$ & 126 & $66 \%$ \\
\hline & Bevacizumab & 866 & $56 \%$ & 65 & $74 \%$ & 437 & $59 \%$ & 0.007 & 37 & $63 \%$ & 235 & $49 \%$ & 92 & $48 \%$ \\
\hline & Anti-EGFR & 364 & $23 \%$ & 0 & $0 \%$ & 36 & $5 \%$ & $\mathrm{NE}$ & 10 & $17 \%$ & 289 & $61 \%$ & 29 & $15 \%$ \\
\hline
\end{tabular}

NE, no estimate; ref, reference.

${ }^{*} p$-Value between KRAS-G12C mt and Other KRAS mt. Significant differences in bold.

with proportions seen in large databases of molecularly tested tumors $(3 \%-7 \%)(12,14,18,36)$.

We were not able to detect any significant or clinically relevant differences in patient characteristics, treatments provided, and outcomes between the 103 KRAS-G12C cases compared to those with other KRAS or NRAS mutations. This contrasts with the results of previous studies claiming differences in several aspects, however, not consistent. Above all, we could not show any differences in response rates, PFS, or OS, especially not when analyzed per treatment group defined as cytotoxics with or without anti-VEGF/anti-EGFR, or curative intent metastasectomy and/or LAT, allowing us to conclude that tumors with this mutation behave like those with other KRAS mutations in mCRC. Any association of KRAS-G12C in smokers, whether current or ex-smokers, versus never smokers could not be verified among mCRC patients, in contrast to lung cancer where KRAS-G12C has been associated with smoking, especially in women $(37,38)$. Salem et al. reported that dMMR status was less common in KRAS-G12C than in other tumors (36), a trend not verified in our study ( $3 \%$ for both). But we had numerically higher dMMR rates in NRAS-mutated (10\%) and BRAF-V600Emutated (29\%) tumors, with the caveat of small patient numbers. KRAS-G12C mutations were mutually exclusive for other KRAS, $N R A S$, and BRAF-V600E mutations in all but one case (with BRAF-V600Emt), which is in line with previous findings (14).

To show differences between rare tumor properties, large and preferably non-selected patient series are required. Most studies exploring the clinical relevance of tumor properties stem from clinical trials, and in this respect, randomized trials are considered most valuable. However, inclusion in trials and particularly in randomized trials means that patient selection can be extensive. We and others have previously reported that
BRAF-V600E mutations are at least twice as common in the background population as in the trial-/hospital-based series (24, $26,39)$, and similarly, dMMR is about twice as common $(26,40)$. When the disease is metastatic, both BRAF-V600E and dMMR mean poor prognosis, and the most probable reason behind these prevalence differences is that patients with these tumors do not frequently manage to be included because of rapidly progressing disease. Comorbidities related to older age may also contribute to the risk. A proportion of $2 \%$ of KRAS-G12C in the populationbased cohorts, including a significant BSC population and higher proportions of treatable patients as in the real-life RAXO-study and in the Finnish population cohorts, where only "treatable" patients were molecularly analyzed, rather indicates that, if anything, the prognosis for this mutation is better than that for other KRAS mutations.

It is very difficult to evaluate the extent of patient selection in different patient materials, although younger age usually indicates more selection since cancers such as CRC are a disease of the elderly. In $\mathrm{mCRC}$, a higher proportion of rightsided colon tumors, more female patients, older age, and more patients with poor performance (and thus fewer patients actively treated) generally indicate less selection. The PRCRC-study and Uppsala region cohorts were created with the intent to minimize selection having a prospective design with efforts to include as many patients as possible and finally to retrospectively identify remaining patients via registries. The entire PRCRC-study and Uppsala region cohorts have, thus, probably identified all (100\%) in vivo diagnosed cases with $\mathrm{mCRC}$. The RAXO data collection has the same intention, but as data are not validated yet, only patients with KRAS-G12C tumors were reported with a nearly $100 \%$ capture in the four regions covering $62 \%$ of the Finnish population. For the molecular analyses, selection will inevitably 
A

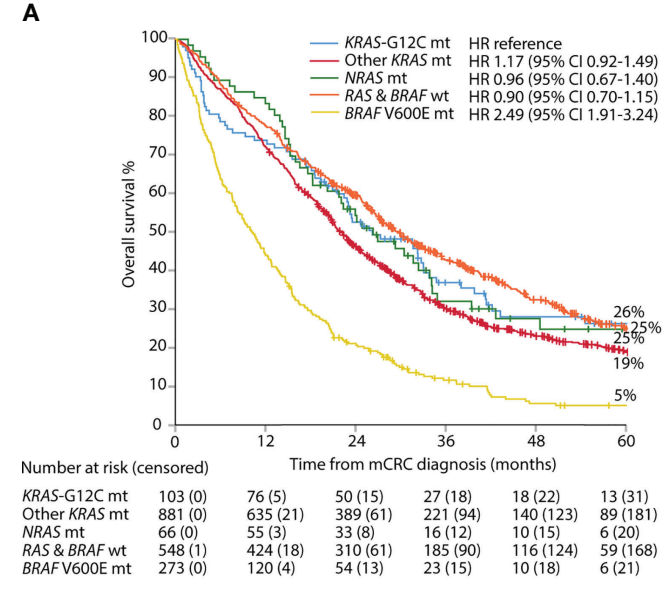

C

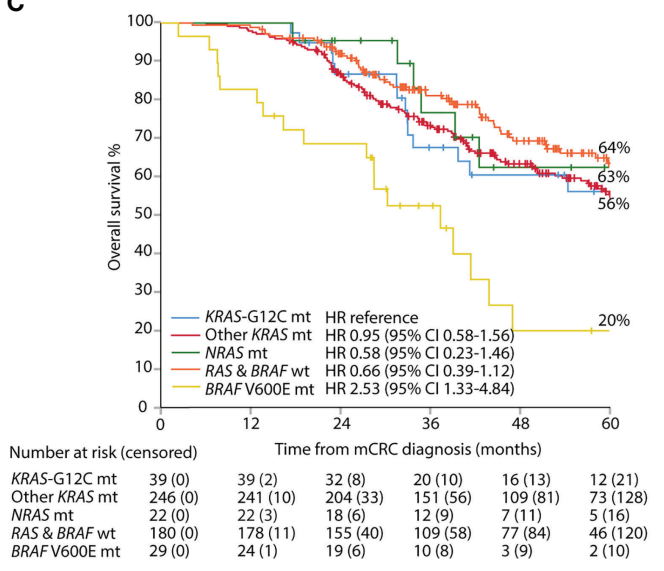

B

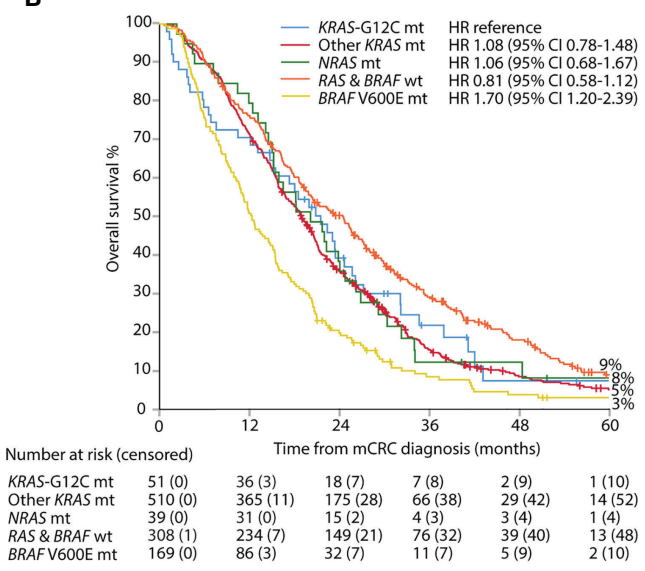

D

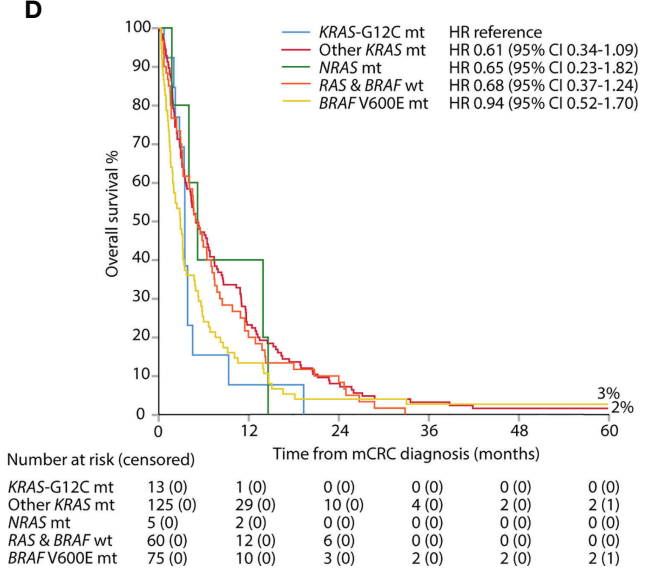

FIGURE 2 | Overall survival per group of mutation: all patients (A), systemic therapy only (B), metastasectomy and/or local ablative therapy (C), and best supportive care (D).

be present since diagnostic materials sufficient for analysis will not always be obtained from the oldest patients and from those with a rapid clinical course. Lack of sufficient tissue for molecular analyses in mCRC is a significant negative prognostic marker (24). Even if a biopsy was aimed at, it was not infrequently insufficient for any analyses more than to confirm the diagnosis of invasive adenocarcinoma. If materials had been obtained from every individual (i.e., also the $17 \%$ with no molecular analyses), it is possible that KRAS-G12C had been even slightly less common and, conversely, BRAF-V600E and dMMR even more frequent.

A weakness of the study is that all molecular tests were done in clinical routine, however, as recommended by ESMO guidelines $(33,34)$. This meant that for patients included early in the Uppsala cohort and in the Finnish population cohort, rare KRAS mutations such as those in codons 117 and 146, NRAS mutations, and $B R A F$ mutations were not covered. This does not invalidate the estimations of the proportions of KRAS-G12C among all patients with mCRC but slightly overestimates the proportion of KRAS-G12 mutations among those with any KRAS mutation. From a scientific point of view, the use of only one technique covering all relevant genes would have been an advantage. However, relying on testing performed in clinical routine could also be looked upon as a strength considering the purpose of the study to explore the presence and behavior of this mutation in relation to others in the background population.

It is legitimate to discuss the relevance of finding the true prevalence of a certain trait in the background population. When it comes to toxic treatments such as chemotherapy and many target drugs, the patients must be at least reasonably well to be treated. Newer drugs may be less toxic and have an entirely different toxicity profile, making it highly relevant to know the distribution also in elderly, frail, and poor-performance-status patients. Immunotherapy for dMMR may be an example of this. The specific drugs presently under development for this mutation may be another example, as, at least in the early phases of development, toxicity profiles are considered "favorable" $(7,8)$.

In conclusion, in these real-life and population-based cohorts reflecting the background population, we did not detect any significant or clinically relevant differences in patient characteristics and tumor outcomes between patients with KRAS-G12C tumors compared to those with other KRAS mutations. The similarities in OS between KRAS-G12C and 
other-KRAS mutant tumors were seen irrespective of if the patients were treated with cytotoxics only or in combination with biologic agents, treated with metastasectomy and/or LAT, or handled with BSC. This contrasts with the results of previous studies mostly claiming worse outcomes in KRAS-G12C patients. Since population-based studies, minimizing patient selection, are more relevant for studies exploring the natural course of any property than clinical trials are, it is our belief that KRAS-G12C tumors behave similarly to all other KRAS- (and NRAS-) mutated tumors. Clinical trials following specific treatment protocols are more relevant when exploring outcomes after specific treatments. We could not detect any indications that the mutations differed when homogeneous treatment groups were analyzed and, thus, also believe that prognosis is not different according to the treatments used today, although our conclusion is based upon a few patients in each treatment group, however, not fundamentally fewer than in most studies having noticed worse survival for the KRAS-G12C mutation group. When specific drugs are developed, as for this mutation, differences in outcome will hopefully emerge.

\section{CONGRESSES}

Presented in part at the European Society for Medical Oncology (ESMO) World Congress on Gastrointestinal Cancer, Barcelona, June 30-July 3, 2021.

\section{DATA AVAILABILITY STATEMENT}

The data collected for this study can be made available to others in de-identified form after all primary and secondary endpoints have been published, in the presence of a data transfer agreement, and if the purpose of use complies with Nordic legislation. Requests for data sharing including a proposal that must be approved by the steering committee should be directed to BG, bengt.glimelius@ igp.uu.se and PO, pia.osterlund@helsinki.fi.

\section{ETHICS STATEMENT}

The studies involving human participants were reviewed and approved by Ethical Board at Bergen, Helsinki, Odense, and Uppsala University Hospitals. The prospective patients/ participants provided their written informed consent to participate in this study.

\section{REFERENCES}

1. Vogelstein B, Papadopoulos N, Velculescu VE, Zhou S, Diaz LA Jr., Kinzler KW. Cancer Genome Landscapes. Science (2013) 339(6127):1546-58. doi: $10.1126 /$ science. 1235122

2. Sorich MJ, Wiese MD, Rowland A, Kichenadasse G, McKinnon RA, Karapetis CS. Extended RAS Mutations and Anti-EGFR Monoclonal Antibody Survival Benefit in Metastatic Colorectal Cancer: A Meta-Analysis of Randomized, Controlled Trials. Ann Oncol (2015) 26(1):13-21. doi: 10.1093/annonc/mdu378

\section{AUTHOR CONTRIBUTIONS}

EO, AR, SK, TK, EH, TM, PH, RK, L-MS, JS, MK, AÅ, RR, HS, PP, LN, TSa, AL, MM, TSj, HI, BG, and PO comprised the steering committee and participated in all phases of the study, including the design or conduct of the study, analyses and interpretation of the data, preparation of the manuscript, and the decision to submit. All authors recruited patients or gathered data for the study. EO and PO did the statistical analyses.

\section{FUNDING}

Finska Läkaresällskapet (2016, 2018, 2019, 2020, 2021, 2022), The Finnish Cancer Foundation (2019-2020, 2021, 2022-2023), The Competitive State Research Financing of the Expert Responsibility Area of Tampere, Turku, Helsinki, Oulu and Kuopio University Hospitals (2016, 2017, 2018, 2019, 2020, 2021, 2022), Tampere and Helsinki University Hospital Research Funds (Tukisäätiö 2019, 2020; OOO 2020), The Sigrid Jusélius Foundation (2017, 2021), and The Swedish Cancer Society $(2016,2019)$ have provided grants. The infrastructure of the RAXO-study, with blood sampling, database, and study nurses, was supported by pharmaceutical companies: Amgen (unrestricted grants, 2012-2020), Lilly (20122017), Merck KGaA (2012-2020), Roche Oy (2012-2020), Sanofi (2012-2017), and Servier (unrestricted grant, 2016-2020). Amgen also partly supported the NGS analysis performed in patients included in the Uppsala region cohort. The funding sources had no role in the design and conduct of the study, collection, analysis and interpretation of the data, or decision to submit the manuscript for publication.

\section{ACKNOWLEDGMENTS}

We would like to thank the RAXO-study and PRCRC-study investigators and study nurses.

\section{SUPPLEMENTARY MATERIAL}

The Supplementary Material for this article can be found online at: https://www.frontiersin.org/articles/10.3389/fonc.2022.826073/ full\#supplementary-material

3. Van Cutsem E, Lenz HJ, Kohne CH, Heinemann V, Tejpar S, Melezinek I, et al. Fluorouracil, Leucovorin, and Irinotecan Plus Cetuximab Treatment and RAS Mutations in Colorectal Cancer. J Clin Oncol (2015) 33(7):692-700. doi: 10.1200/JCO.2014.59.4812

4. Tran E, Ahmadzadeh M, Lu YC, Gros A, Turcotte S, Robbins PF, et al. Immunogenicity of Somatic Mutations in Human Gastrointestinal Cancers. Science (2015) 350(6266):1387-90. doi: 10.1126/science.aad1253

5. Hobbs GA, Der CJ, Rossman KL. RAS Isoforms and Mutations in Cancer at a Glance. J Cell Sci (2016) 129(7):1287-92. doi: 10.1242/jcs.182873 
6. Stahler A, Heinemann V, Ricard I, von Einem JC, Giessen-Jung C, Westphalen CB, et al. Current Treatment Options in RAS Mutant Metastatic Colorectal Cancer Patients: A Meta-Analysis of 14 Randomized Phase III Trials. J Cancer Res Clin Oncol (2020) 146(8):2077-87. doi: 10.1007/ s00432-020-03290-y

7. Hong DS, Fakih MG, Strickler JH, Desai J, Durm GA, Shapiro GI, et al. KRAS (G12C) Inhibition With Sotorasib in Advanced Solid Tumors. N Engl J Med (2020) 383(13):1207-17. doi: 10.1056/NEJMoa1917239

8. Wang C, Fakih M. Targeting KRAS in Colorectal Cancer. Curr Oncol Rep (2021) 23(3):28. doi: 10.1007/s11912-021-01022-0

9. Smith G, Bounds R, Wolf H, Steele RJ, Carey FA, Wolf CR. Activating K-Ras Mutations Outwith 'Hotspot' Codons in Sporadic Colorectal Tumours Implications for Personalised Cancer Medicine. Br J Cancer (2010) 102 (4):693-703. doi: 10.1038/sj.bjc.6605534

10. Vaughn CP, Zobell SD, Furtado LV, Baker CL, Samowitz WS. Frequency of KRAS, BRAF, and NRAS Mutations in Colorectal Cancer. Genes Chromosomes Cancer (2011) 50(5):307-12. doi: 10.1002/gcc.20854

11. Modest DP, Ricard I, Heinemann V, Hegewisch-Becker S, Schmiegel W, Porschen R, et al. Outcome According to KRAS-, NRAS- and BRAF-Mutation as Well as KRAS Mutation Variants: Pooled Analysis of Five Randomized Trials in Metastatic Colorectal Cancer by the AIO Colorectal Cancer Study Group. Ann Oncol (2016) 27(9):1746-53. doi: 10.1093/annonc/mdw261

12. Serebriiskii IG, Connelly C, Frampton G, Newberg J, Cooke M, Miller V, et al. Comprehensive Characterization of RAS Mutations in Colon and Rectal Cancers in Old and Young Patients. Nat Commun (2019) 10(1):3722. doi: 10.1038/s41467-019-11530-0

13. Schirripa M, Nappo F, Cremolini C, Salvatore L, Rossini D, Bensi M, et al. KRAS G12C Metastatic Colorectal Cancer: Specific Features of a New Emerging Target Population. Clin Colorectal Cancer (2020) 19(3):219-25. doi: 10.1016/j.clcc.2020.04.009

14. Araujo LH, Souza BM, Leite LR, Parma SAF, Lopes NP, Malta FSV, et al. Molecular Profile of KRAS G12C-Mutant Colorectal and Non-Small-Cell Lung Cancer. BMC Cancer (2021) 21(1):193. doi: 10.1186/s12885-021-07884-8

15. Henry JT, Coker O, Chowdhury S, Shen JP, Morris VK, Dasari A, et al. Comprehensive Clinical and Molecular Characterization of KRAS (G12C)Mutant Colorectal Cancer. JCO Precis Oncol (2021) 5:613-21. doi: 10.1200/ PO.20.00256

16. Chida K, Kotani D, Masuishi T, Kawakami T, Kawamoto Y, Kato K, et al. The Prognostic Impact of KRAS G12C Mutation in Patients with Metastatic Colorectal Cancer: A Multicenter Retrospective Observational Study. Oncologist (2021) 26(10):845-53. doi: 10.1002/onco.13870

17. Giampieri R, Lupi A, Ziranu P, Bittoni A, Pretta A, Pecci F, et al. Retrospective Comparative Analysis of KRAS G12C vs. Other KRAS Mutations in mCRC Patients Treated With First-Line Chemotherapy Doublet + Bevacizumab. Front Oncol (2021) 11:736104. doi: 10.3389/fonc.2021.736104

18. Li N, Li D, Du Y, Su C, Yang C, Lin C, et al. Overexpressed PLAGL2 Transcriptionally Activates Wnt6 and Promotes Cancer Development in Colorectal Cancer. Oncol Rep (2019) 41(2):875-84. doi: 10.3892/or.2018.6914

19. Patelli G, Tosi F, Amatu A, Mauri G, Curaba A, Patane DA, et al. Strategies to Tackle RAS-Mutated Metastatic Colorectal Cancer. ESMO Open (2021) 6 (3):100156. doi: 10.1016/j.esmoop.2021.100156

20. Ottaiano A, Normanno N, Facchini S, Cassata A, Nappi A, Romano C, et al. Study of Ras Mutations' Prognostic Value in Metastatic Colorectal Cancer: STORIA Analysis. Cancers (Basel) (2020) 12(7):1919. doi: 10.3390/ cancers 12071919

21. Neumann J, Zeindl-Eberhart E, Kirchner T, Jung A. Frequency and Type of KRAS Mutations in Routine Diagnostic Analysis of Metastatic Colorectal Cancer. Pathol Res Pract (2009) 205(12):858-62. doi: 10.1016/j.prp. 2009.07.010

22. Gil Ferreira C, Aran V, Zalcberg-Renault I, Victorino AP, Salem JH, Bonamino $\mathrm{MH}$, et al. KRAS Mutations: Variable Incidences in a Brazilian Cohort of 8,234 Metastatic Colorectal Cancer Patients. BMC Gastroenterol (2014) 14:73. doi: 10.1186/1471-230X-14-73

23. Modest DP, Brodowicz T, Stintzing S, Jung A, Neumann J, Laubender RP, et al. Impact of the Specific Mutation in KRAS Codon 12 Mutated Tumors on Treatment Efficacy in Patients With Metastatic Colorectal Cancer Receiving Cetuximab-Based First-Line Therapy: A Pooled Analysis of Three Trials. Oncology (2012) 83(5):241-7. doi: 10.1159/000339534
24. Sorbye H, Dragomir A, Sundstrom M, Pfeiffer P, Thunberg U, Bergfors M, et al. High BRAF Mutation Frequency and Marked Survival Differences in Subgroups According to KRAS/BRAF Mutation Status and Tumor Tissue Availability in a Prospective Population-Based Metastatic Colorectal Cancer Cohort. PloS One (2015) 10(6):e0131046. doi: 10.1371/journal.pone.0131046

25. Aasebo KO, Dragomir A, Sundstrom M, Mezheyeuski A, Edqvist PH, Eide GE, et al. Consequences of a High Incidence of Microsatellite Instability and BRAF-Mutated Tumors: A Population-Based Cohort of Metastatic Colorectal Cancer Patients. Cancer Med (2019) 8(7):3623-35. doi: 10.1002/cam4.2205

26. Nunes L, Aasebo K, Mathot L, Ljungstrom V, Edqvist PH, Sundstrom M, et al. Molecular Characterization of a Large Unselected Cohort of Metastatic Colorectal Cancers in Relation to Primary Tumor Location, Rare Metastatic Sites and Prognosis. Acta Oncol (2020) 59(4):417-26. doi: 10.1080/ 0284186X.2019.1711169

27. Osterlund P, Salminen T, Soveri LM, Kallio R, Kellokumpu I, Lamminmaki A, et al. Repeated Centralized Multidisciplinary Team Assessment of Resectability, Clinical Behavior, and Outcomes in 1086 Finnish Metastatic Colorectal Cancer Patients (RAXO): A Nationwide Prospective Intervention Study. Lancet Reg Health Eur (2021) 3:100049. doi: 10.1016/j.lanepe. 2021.100049

28. Isoniemi H, Uutela A, Nordin A, Lantto E, Kellokumpu I, Ovissi A, et al. Centralized Repeated Resectability Assessment of Patients With Colorectal Liver Metastases During First-Line Treatment: Prospective Study. Br J Surg (2021) 108(7):817-25. doi: 10.1093/bjs/znaa145

29. Sorbye H, Pfeiffer P, Cavalli-Bjorkman N, Qvortrup C, Holsen MH, WentzelLarsen T, et al. Clinical Trial Enrollment, Patient Characteristics, and Survival Differences in Prospectively Registered Metastatic Colorectal Cancer Patients. Cancer (2009) 115(20):4679-87. doi: 10.1002/cncr.24527

30. Glimelius B, Melin B, Enblad G, Alafuzoff I, Beskow A, Ahlstrom H, et al. UCAN: A Prospective Longitudinal Collection of Biomaterials and Clinical Information From Adult Cancer Patients in Sweden. Acta Oncol (2018) 57 (2):187-94. doi: 10.1080/0284186X.2017.1337926

31. Pahlman L, Bohe M, Cedermark B, Dahlberg M, Lindmark G, Sjodahl R, et al. The Swedish Rectal Cancer Registry. Br J Surg (2007) 94(10):1285-92. doi: 10.1002/bjs.5679

32. Osterman E, Hammarstrom K, Imam I, Osterlund E, Sjoblom T, Glimelius B. Completeness and Accuracy of the Registration of Recurrences in the Swedish Colorectal Cancer Registry (SCRCR) and an Update of Recurrence Risk in Colon Cancer. Acta Oncol (2021) 60(7):842-9. doi: 10.1080/0284186X.2021.1896033

33. Schmoll HJ, Van Cutsem E, Stein A, Valentini V, Glimelius B, Haustermans $\mathrm{K}$, et al. ESMO Consensus Guidelines for Management of Patients With Colon and Rectal Cancer. A Personalized Approach to Clinical Decision Making. Ann Oncol (2012) 23(10):2479-516. doi: 10.1093/annonc/mds236

34. Van Cutsem E, Cervantes A, Adam R, Sobrero A, Van Krieken JH, Aderka D, et al. ESMO Consensus Guidelines for the Management of Patients With Metastatic Colorectal Cancer. Ann Oncol (2016) 27(8):1386-422. doi: 10.1093/annonc/mdw235

35. Sundstrom M, Edlund K, Lindell M, Glimelius B, Birgisson H, Micke P, et al. KRAS Analysis in Colorectal Carcinoma: Analytical Aspects of Pyrosequencing and Allele-Specific PCR in Clinical Practice. BMC Cancer (2010) 10:660. doi: 10.1186/1471-2407-10-660

36. Salem M, El-Refai S, Sha W, Grothey A, Puccini A, George T, et al. Characterization of KRAS Mutation Variants and Prevalence of KRASG12C in Gastrointestinal Malignancies. Ann Oncol (2021) 32:S218. doi: 10.1016/j.annonc.2021.05.007

37. Riely GJ, Kris MG, Rosenbaum D, Marks J, Li A, Chitale DA, et al. Frequency and Distinctive Spectrum of KRAS Mutations in Never Smokers With Lung Adenocarcinoma. Clin Cancer Res (2008) 14(18):5731-4. doi: 10.1158/10780432.CCR-08-0646

38. Dogan S, Shen R, Ang DC, Johnson ML, D’Angelo SP, Paik PK, et al. Molecular Epidemiology of EGFR and KRAS Mutations in 3,026 Lung Adenocarcinomas: Higher Susceptibility of Women to Smoking-Related KRAS-Mutant Cancers. Clin Cancer Res (2012) 18(22):6169-77. doi: 10.1158/1078-0432.CCR-11-3265

39. Algars A, Sundstrom J, Lintunen M, Jokilehto T, Kytola S, Kaare M, et al. EGFR Gene Copy Number Predicts Response to Anti-EGFR Treatment in RAS Wild Type and RAS/BRAF/PIK3CA Wild Type Metastatic Colorectal Cancer. Int J Cancer (2017) 140(4):922-9. doi: 10.1002/ijc.30507 
40. Aasebo K, Dragomir A, Sundstrom M, Mezheyeuski A, Edqvist PH, Eide GE, et al. CDX2: A Prognostic Marker in Metastatic Colorectal Cancer Defining a Better BRAF Mutated and a Worse KRAS Mutated Subgroup. Front Oncol (2020) 10:8. doi: 10.3389/fonc.2020.00008

Conflict of Interest: EO, AR, SK, TK, EH, TM, PH, RK, L-MS, JS, MK, A , RR, $\mathrm{PP}, \mathrm{TSa}, \mathrm{AL}, \mathrm{MM}, \mathrm{HI}$, and $\mathrm{PO}$ report institutional research funding from Eli Lilly, Merck KGaA, Nordic Drugs, Roche Oy, and Sanofi or unrestricted grants from Amgen and Servier, during the conduct of the study. EO, LN, TSj, and BG report unrestricted grants from Amgen for molecular analysis in the Uppsala region cohort. EO, AR, SK, TK, EH, TM, PH, RK, L-MS, JS, MK, AÅ, RR, HS, PP, TSa, $\mathrm{AL}, \mathrm{MM}, \mathrm{HI}$, and $\mathrm{PO}$ report grants, personal fees, or non-financial support from AbbVie, Amgen, Astra-Zeneca, Bayer, Celgene, Eli Lilly, Eisai, Erytech Pharma, Incyte, Fresenius, Jansen-Cilag, Merck, MSD, Nordic Drugs, Nutricia, PierreFabre, Roche, Sanofi, Servier, Sobi, or Varian.
Publisher's Note: All claims expressed in this article are solely those of the authors and do not necessarily represent those of their affiliated organizations, or those of the publisher, the editors and the reviewers. Any product that may be evaluated in this article, or claim that may be made by its manufacturer, is not guaranteed or endorsed by the publisher.

Copyright (C) 2022 Osterlund, Ristimäki, Kytölä, Kuopio, Heervä, Muhonen, Halonen, Kallio, Soveri, Sundström, Keinänen, Ålgars, Ristamäki, Sorbye, Pfeiffer, Nunes, Salminen, Lamminmäki, Mäkinen, Sjöblom, Isoniemi, Glimelius and Osterlund. This is an open-access article distributed under the terms of the Creative Commons Attribution License (CC BY). The use, distribution or reproduction in other forums is permitted, provided the original author(s) and the copyright owner(s) are credited and that the original publication in this journal is cited, in accordance with accepted academic practice. No use, distribution or reproduction is permitted which does not comply with these terms. 